\title{
Multi-diagnostic analysis of plasma filaments in the
}

\section{island divertor}

\author{
S. Zoletnik ${ }^{1}$, G. Anda ${ }^{1}$, C. Biedermann ${ }^{2}$, A, D. Carralero ${ }^{3}$, G. Cseh $^{1}$, D. Dunai ${ }^{1}$, C. \\ Killer $^{2}$, G. Kocsis ${ }^{1}$, A. Krämer-Flecken ${ }^{4}$, M. Otte ${ }^{2}$, B. Shanahan ${ }^{2}$, T. Szepesi ${ }^{1}$, M. \\ Vecsei $^{1}$, L. Zsuga ${ }^{1}$, W7-X Team ${ }^{2}$ \\ ${ }^{1}$ Wigner Research Centre for Physics, P.O.Box 49, H-1525, Budapest, Hungary \\ ${ }^{2}$ Max-Planck-Insitut für Plasmaphysik, Wendelsteinstr 1, D-17491 Greifswald, Germany \\ ${ }^{3}$ Laboratorio Nacional de Fusión, 28040 Madrid, Spain \\ ${ }^{4}$ Institute for Climate and Energy Research-Plasma Physics, Forschungszentrum Jülich, D-52425 Jülich, Germany \\ E-mail: zoletnik.sandor@wigner.mta.hu \\ Received $\mathrm{xxxxxx}$ \\ Accepted for publication $\mathrm{xxxxxx}$ \\ Published $\operatorname{xxxxxx}$
}

\section{Abstract}

Filaments or blobs are well known structures in turbulence in magnetic fusion devices, they are considered to be the major cross-transport channel in the scrape off layer. They originate at the last closed magnetic flux surface and propagate out on the low field side of the toroidal devices due to polarization in the curved magnetic field. The Wendelstein 7-X stellarator has complex three-dimensional magnetic field structure and additionally the plasma is bounded by a chain of magnetic islands, forming an island divertor. After first observation of filaments in Wendelstein 7-X with video cameras a multi-diagnostic study is presented in this paper to reveal their 3D structure and dynamics. Filaments are seen to born at the edge and, at least in some case, seen to extent to up to 4 toroidal turns. After moving radially out a few $\mathrm{cm}$ they enter the edge island. Here they disappear from the equatorial plane and about 200 microsecond later reappear on the outboard side of the island. A long-wavelength $(\sim 20-30 \mathrm{~cm})$ quasi coherent mode is observed in both regions where filaments appear. Similarities and differences between the filaments seen in W7$\mathrm{X}$ and other devices are discussed. Possible explanations for this strange radial propagation are considered together with the likely role of filaments in the edge and island density profile. 


\section{Introduction}

Filaments or blobs[1] are well known phenomena in magnetically confined plasmas. They are unipolar plasma structures localized to $\mathrm{mm}-\mathrm{cm}$ size across the magnetic field while having long, up to several 10 meter, extent along the magnetic field lines. In toroidal devices (tokamaks and stellarators) their birth location is in the edge plasma, close to the last closed flux surface. Although there is no final agreement on details of blob generation, 2D models suggests that they are produced in the nonlinear phase of the interchange instability on the outboard side of the torus. In its nonlinear growth phase the instability generates filaments which detach from the main plasma. Curvature and gradient drift causes charge separation in the filament and the resulting electric field moves it with an ExB drift velocity against the gradient of the magnetic field. Filaments have been extensively studied in tokamak devices where they carry substantial amount of particles and energy from the edge plasma into the Scrape Off Layer (SOL), where magnetic field lines cross material surfaces. In the SOL they are considered to be the major cross-field transport mechanism.

Connection to the material surfaces can affect the electric field in the filament. Two situations are distinguished. In the 'sheath-limited' regime polarization is reduced by current flowing along the filament (magnetic field line) and closing on material surfaces, thus the electric field and the blob velocity is reduced. Under certain conditions (e.g. at high density and/or long connection length) B-perpendicular currents limit the polarization and the 'inertial regime' sets in. Transition between the two regimes changes the blob velocity thus affecting radial transport in the SOL and changing the density profile. A recent study[2] showed that the transition to the inertial filament regime and the density shoulder are often linked, therefore blob dynamics are considered to be an important element in setting the SOL conditions. 


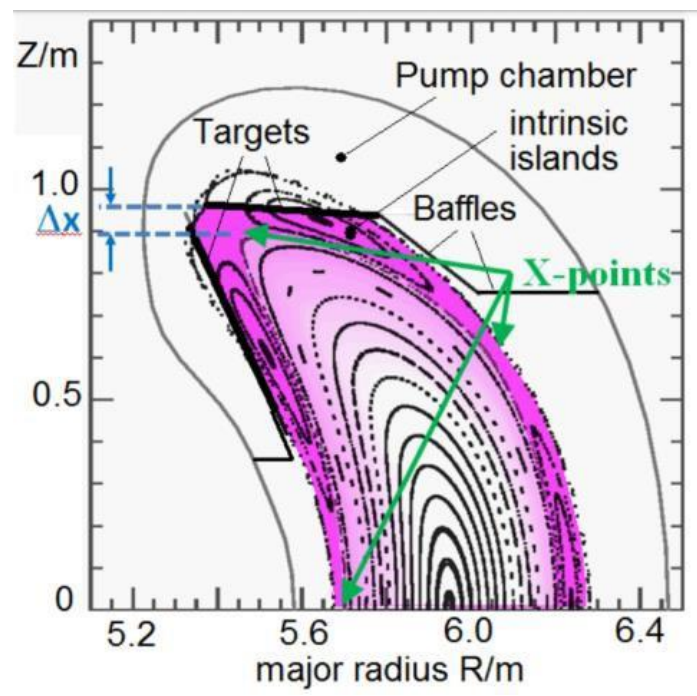

Figure 1. Section of one half of the plasma with Poincare plot of vacuum magnetic field lines in the standard configuration of Wendelstein 7-X.[10]

The dynamics of filaments are considered mostly understood in the quasi-2D situations where the curvature and shearing of magnetic field lines changes only moderately along the filament. This is the situation in tokamak configurations where the plasma is bounded by limiters and in poloidal divertor configurations far outside the separatrix in the far SOL region. Around the separatrix, and especially close to the X-point, the strong magnetic shear distorts the filaments[3][4] and a 3D treatment becomes necessary[5].

Although blobs were mostly studied in tokamaks there are measurement results from stellarators and lowtemperature toroidal devices as well[1], where similar features were found. In the context of the present paper the comparison between ASDEX and Wendelstein 7-AS (W7-AS)[6], should be explicitly mentioned where basic filament properties were found to agree.

This paper describes the first multi-diagnostic observations of filaments in a different 3D configuration, the edge and island divertor of the Wendelstein 7-X (W7-X) stellarator. Although W7-AS was the predecessor experiment to W7-X filaments in the island divertor of W7-AS were not studied. Therefore the W7-X experiments offer the first possibility to study filaments in this special configuration.

The paper is organized as follows. In Section 2 relevant differences between the W7-X and tokamak configurations are considered and some initial modelling results illustrate their consequences. Section 3 describes the diagnostics which were used to obtain the experimental results in Section 4. In the Discussion some interpretation possibilities and open questions are presented and the results are summarized in a brief Conclusions.

\section{Differences between Wendelstein 7-X and tokamak configuration}

Wendelstein 7X is an optimized, low shear, modular stellarator built from 5 identical toroidal modules. 
The machine started operation in 2016 in limiter configuration[7] and continued in 2017-18 using various inertially cooled island divertor configurations[8]. In the 'standard' configuration the rotational transform at the plasma edge is close to 1 and the core plasma is surrounded by a chain of 5 natural magnetic islands, each connecting onto itself after one toroidal turn. The islands follow the magnetic field lines therefore, unlike in tokamaks, the rotational transform changes smoothly across the island separatrix.

The islands are intersected by divertor plates at various toroidal locations, forming an island divertor[8][9][10] as shown in Figure 1. In this configuration the connection length from the equatorial plane to the divertor plates is much longer than in tokamaks[9], up to $1000 \mathrm{~m}$ and dropping to about $50 \mathrm{~m}$ on the outboard side of the island. Additionally, a closed flux bundle is present around the o-point of the island in the vacuum field calculation. This strongly varying connection length may cause transitions to and back between inertial and sheath limited regimes resulting in strong variations in the filament polarization, and consequently its radial velocity. It has to be noted that plasma pressure and bootstrap current can slightly $(\sim 1 \mathrm{~cm})$ shift the location of the magnetic islands, but in this paper these effects are not taken into account.

The rotational transform at the inner island separatrix is about 0.98 . Therefore, field lines starting around the separatrix at the outboard equatorial plane end up about $25-30 \mathrm{~cm}$ poloidal distance after one toroidal turn. This means that for perturbations having a poloidal wavelength in the $10 \mathrm{~cm}$ range the plasma cannot be considered infinitely long along the magnetic field lines and a 3D treatment is inevitable.

Another feature of $\mathrm{W} 7-\mathrm{X}$ is the 3D geometry where field line curvature is strongly changing along field lines, thus affecting the polarization currents and the electric field in the filaments.

\section{Theoretical considerations}

First modeling[11] of seeded filaments in realistic W7-X geometry (without islands and radial electric field) indicated that field line curvature direction and magnitude changes considerably along field lines, even within a module extending to 72 degree toroidal angle, thus charge separation currents are also modulated. However, conductivity along field lines is high and these local variations are efficiently equilibrated by parallel currents. The resulting mean polarisation is such that the ExB velocity of the filament is directed outward. The modulation of this velocity along the field line was found to be much less than $1 \%$, and therefore the filament shape is not distorted. This way outward propagating filaments are expected in W7-X, similarly to tokamaks.

\section{Diagnostics used in this study}

During the first limiter campaign of W7-X marked filament activity was observed in video camera images[12]. The W7-X overview camera system[13] consists of 10 tangential views, most of them equipped with EDICAM cameras providing overview image at typically $100 \mathrm{~Hz}$ frame rate and in parallel small 
regions of interest running at 5-10 kHz. Although signature of turbulence was observed in these cameras[14] in the present study we use the tangential view (module $2, \varphi \approx 80 \mathrm{deg}$ ) equipped with an image guide (800x1000 individual fibers) attached to a Photron SA-X2 fast framing camera located at port AEQ21.The camera was running at $30-90 \mathrm{kHz}$ frame rate while viewing the whole plasma. Although the camera pixels record light intensity integrated along a straight line through the plasma most of the intensity comes from a narrow radial region at the edge, therefore the cameras do not provide radial information. The light intensity is also modulated toroidally, especially at the divertor, therefore exact localization of the radiation source is difficult to quantify.

To obtain radial information at the plasma edge the Alkali Beam Emission Spectroscopy (ABES) diagnostic[15] is used. It injects a $50 \mathrm{kV}$ neutral Sodium beam[15] in module 2, $\varphi=72$ degrees in the equatorial plane through the o-point of the island divertor in the standard configuration. The beam particles are excited by collisions with plasma electrons and ions and emit light at the Sodium doublet line ( $589 \mathrm{~nm})$ when they return to ground state. The emitted light is measured by a 40-channel detector system viewing from the vertical direction and resolving the light profile into 40 radial locations, with a resolution of 0.5 $\mathrm{cm}$. The temporal resolution is set by the $500 \mathrm{kHz}$ bandwidth and $2 \mathrm{MHz}$ sampling rate. The diameter of the beam is about $2 \mathrm{~cm}$, therefore the diagnostic is sensitive to perturbations having larger than $2 \mathrm{~cm}$ poloidal size.

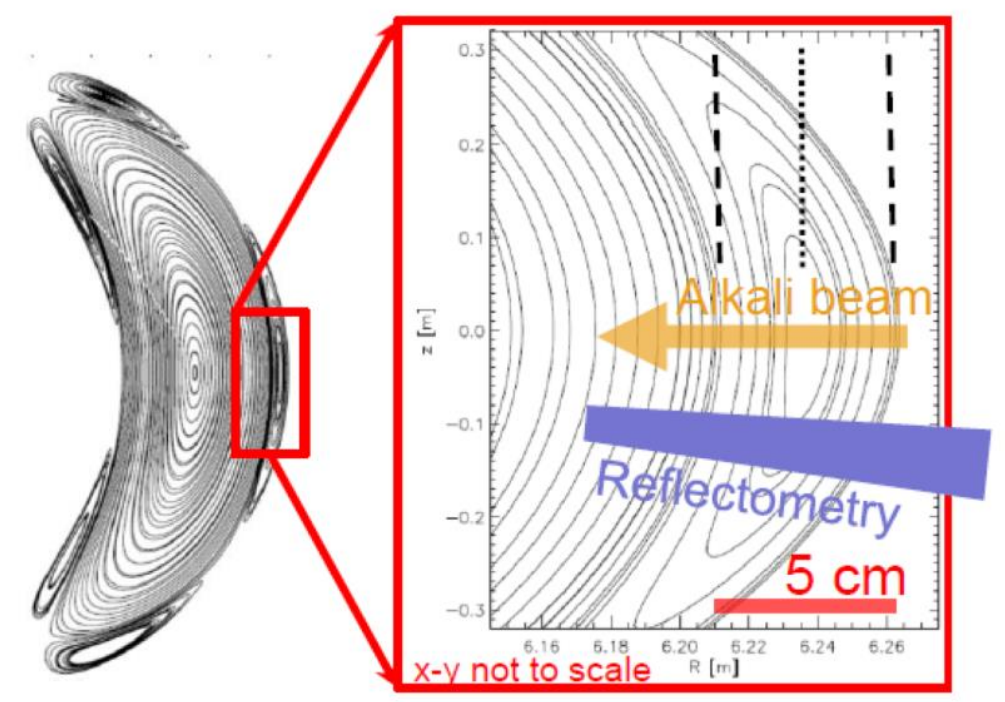

Figure 2. Measurement lines of the ABES and CR diagnostic marked on flux surfaces in the standard configuration. The dashes lines mark the island separatrix radial locations, the dotted line is the island opoint radius.

At low edge density $\left(\leq 3 \times 10^{19} \mathrm{~m}^{-3}\right)$ the light intensity is roughly proportional to the local plasma density as beam ionization is low and the lifetime of the excited Sodium state is about the same as the atom 
flight time between two observation channels. Under these conditions the beam light signal serves as a proxy for the local density in the turbulence analysis. At higher densities or deeper in the plasma beam ionization becomes important and the light signal cannot be considered as a function of local density. On the other hand, this part enables calculation of the absolute plasma density distribution along the beam. Ideally turbulence studies would be done on the reconstructed density profile. At present the reconstruction algorithm is slow and, being a highly nonlinear process, prone to errors. Therefore, the light intensity was used for turbulence studies and high density cases were omitted. A fast reconstruction method for turbulence studies is under development[17]. Under some conditions the density profile exhibits a maximum at the opoint of the island, which is the subject of ongoing investigations.

To gain some poloidal information the Correlation Reflectometer[18] (CR) is used as it is located in the same toroidal angle as the Sodium beam, about $12 \mathrm{~cm}$ below the equatorial plane. This device launches an o-mode polarized microwave beam into the plasma which is reflected from the critical density layer. The complex IQ signal (sine and cosine component) of the reflected beam is measured by 4 antennae forming a poloidal array. The poloidal separation between the uppermost and lowermost measurement point is $40-50$ $\mathrm{mm}$ in the edge and island region. The microwave frequency can be scanned in discrete steps (5-20 ms), this way the reflection layer moves between the outer edge of the island and the edge plasma, somewhat inside the separatrix (see Fig. 2). The measurement location along the beam path is determined from knowledge of the density profile either from the Thomson scattering or the ABES diagnostic. Although a peak in the density profile is often observed at the o-point of the island the density profile is considered to be monotonic along the CR measurement path (as it misses the o-point) and thus measurements cover a continuous radial range. Correlation studies between different antenna complex signals reveal the poloidal flow velocity of turbulence[19]. The relative locations of the ABES and CR diagnostics are shown in Fig. 2.

The above three diagnostics measure close to each other or, in the case of the video camera, view an area where field lines originating from ABES and CR pass by. Therefore, correlation calculations were done to explore the 3D structure of filaments. The correlation function is defined as

$$
C(\tau)=\frac{\int S_{r e f}(t) S(t+\tau) d t}{\sqrt{C_{S}(0) C_{r e f}(0)}}
$$

where $S_{\text {ref }}$ is called the reference signal and we calculate the correlation between $S$ and the reference signal. $C_{S}(0)$ and $C_{r e f}(0)$ are the autocorrelation function at time lag 0 of $S$ and $S_{r e f}$, respectively. In this notation maximum correlation at positive time lag means the reference signal leads.

Some comparison was also done with Langmuir probe measurements[20] performed with reciprocating probe head. As this diagnostic is located roughly the opposite side of the torus and field lines do not connect it with the above diagnostics, no correlation calculations were attempted.

It has to be noted that all of the above diagnostics have spatial resolution in the $\mathrm{cm}$ scale, therefore 
measure the long-wavelength part of the spectrum. Small scale structures were measured with the reciprocating Langmuir probe system[20].

\section{Experimental results}

Low and intermediate density (line density $\leq 3 \times 10^{19} \mathrm{~m}^{-2}$ ) discharges were studied as in this case the alkali beam attenuation is small in the island region. In most analyses (discharge 20181018.003) the standard configuration called "EJM+252" was used at +2.5 T toroidal magnetic field and $3 \mathrm{MW}$ electron cyclotron heating $(\mathrm{ECRH})$, at line density $3 \times 10^{19} \mathrm{~m}^{-2}$. Radially elongated structures are observed in the ABES light profile on the outboard side of the island and also to some extent in the edge plasma as shown in Figure 3.
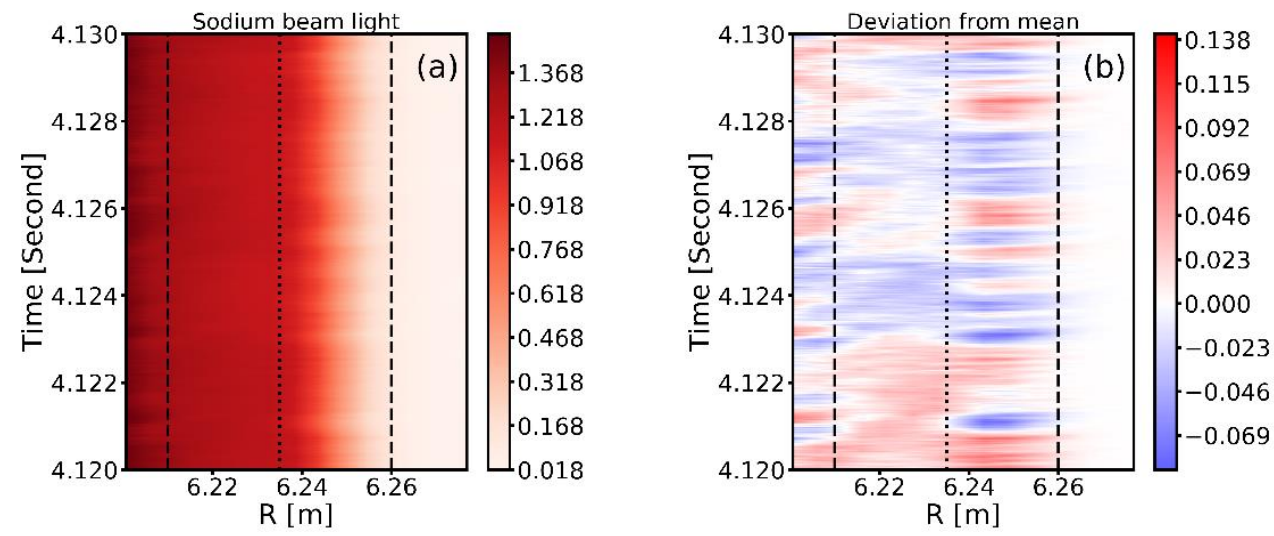

Figure 3. Temporal evolution of the Sodium beam light emission (in arbitrary units) along the beam in discharge 20181018.003 (a) and the same data by subtracting the temporal average from each signal(b).

\section{Power spectra and intermittency}

Three representative autopower spectra of ABES signals are shown in Figure 4. The ABES light detectors have a broadband noise dominated by photon statistics. The approximate noise spectrum is marked by dashed line. Two features are prominent in the spectrum: a broad part extending to about $10 \mathrm{kHz}$ and quasicoherent (QC) peaks in the $10-100 \mathrm{kHz}$ range. At the island o-point only low frequency fluctuations are present but in the edge plasma and the island outboard side similar QC peaks appear. The same QC structures were observed with reflectometry[19]. The reciprocating probe also found strong fluctuations in the outboard side of the island but extending to higher frequencies[20], which can be attributed to the finer spatial resolution and lower noise level of the probes. Coherency between neighbouring ABES channels in this region also shows fluctuations extending to $100 \mathrm{kHz}$. 

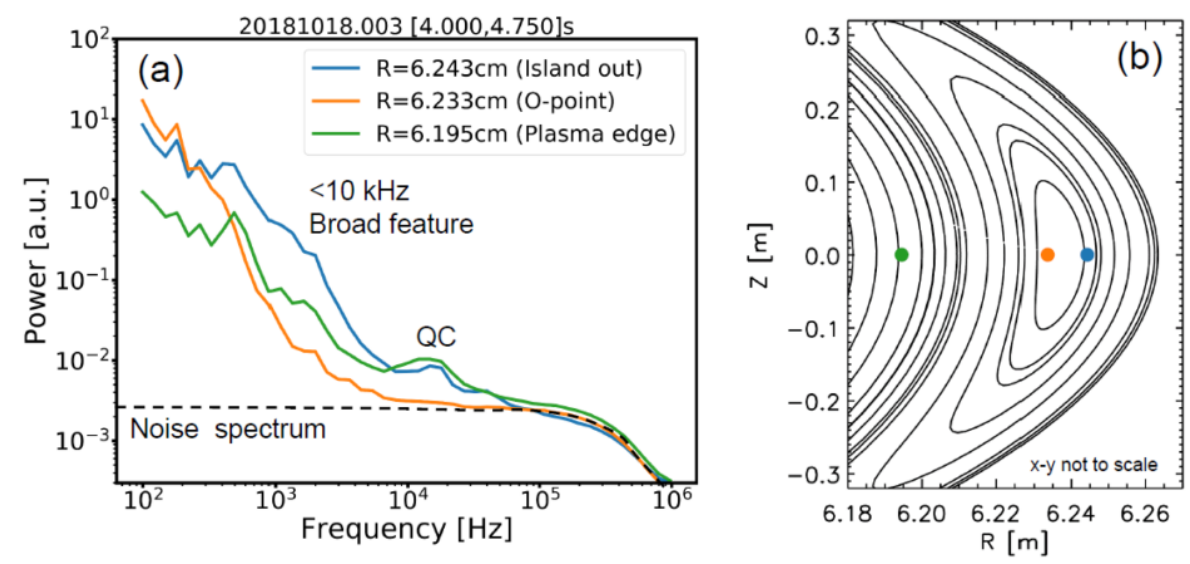

Figure 4. (a) Autopower spectra of ABES signals at the edge plasma, island o-point and the outboard side of the island. The measurement points are marked by the same colour on the flux surface plot (b).

At $1 \mathrm{kHz}$ and a few hundred $\mathrm{Hz}$ quasi-periodic fluctuations exist[21][22] which are seen globally in the machine, while the broadband feature in the 1-10 kHz range is local. These global phenomena are not considered hear.

The relative fluctuation amplitude in the $1 \mathrm{kHz}-10 \mathrm{kHz}$ frequency range in the ABES light signal is up to $5 \%$ on the island outboard side and about $1 \%$ in the edge plasma. These values are considerably lower than the relative fluctuation amplitudes determined by the probe system $(>50 \%[20])$ which can partly be the result of the different spatial resolution[23]. Additionally, especially in the plasma edge, the measured fluctuation amplitude is affected by beam attenuation as described in Ref [24].

The power spectrum of the $1-10 \mathrm{kHz}$ feature is similar to the one measured with BES in the SOL of KSTAR[23] where the spatial resolution is also in the $\mathrm{cm}$ scale. There QC modes regularly appear in the edge plasma as well. The comparison with probe measurements in the KSTAR case showed[23], that the lower frequency spectra and lower fluctuation amplitude compared to the probe measurements are mainly the consequence of the coarser spatial resolution of the BES diagnostic.

Intermittency is a characteristic feature of SOL filaments in tokamaks. In contrast to this individual W7$\mathrm{X}$ ABES signals do not show clearly identifiable events and both positive and negative perturbations are observed. In spatiotemporal plots like Fig 3(b) radially correlated, outward propagating structures can be identified with both positive and negative amplitude. Probe ion saturation signals exhibit some microsecond-scale individual positive pulses on the island outboard side[20], but at lower frequencies no events can be observed.

\section{Radial structure}

The radial structure of fluctuations along the ABES beamline were studied by filtering the signals for 
various frequency bands and calculating cross-correlations as shown in Figure 5. This plot concentrates on the broadband fluctuations between 1.6-10 kHz. To avoid ringing at sharp frequency cutoff first order recursive filters are used on both band limits. When the reference point of the correlation calculation is in the outboard side of the island (Figure 5a), outward propagating structures are observed. The radial velocity is about $400 \mathrm{~m} / \mathrm{s}$ with slowing tendency towards the wall. The slowing down starts at about $6.24 \mathrm{~m}$ where the connection length to the divertor plates drops from few hundred to about $50 \mathrm{~m}$ [20]. When the reference point is in the edge plasma just inside the island inner separatrix (Figure 5b) similar correlation time structures are observed as in the outboard side of the island with some tendency for outward propagation. Interestingly, correlation disappears just inside the island but a significant correlation exists to the outboard side structures with about $200 \mu$ s time lag. At about $500 \mu$ s time lag correlation appears at a large area of the island inboard side as if the density carried by the filaments was deposited there.

The island position is calculated for the ideal vacuum field case. Mechanical distortions of the coil system due to mechanical loads, finite plasma pressure and bootstrap current affect the island position, therefore the precision of the island position calculation is assessed to be about $1 \mathrm{~cm}$. However, reflectometry measurements[19] show a shear layer at the separatrix confirming the validity of the separatrix position calculation.
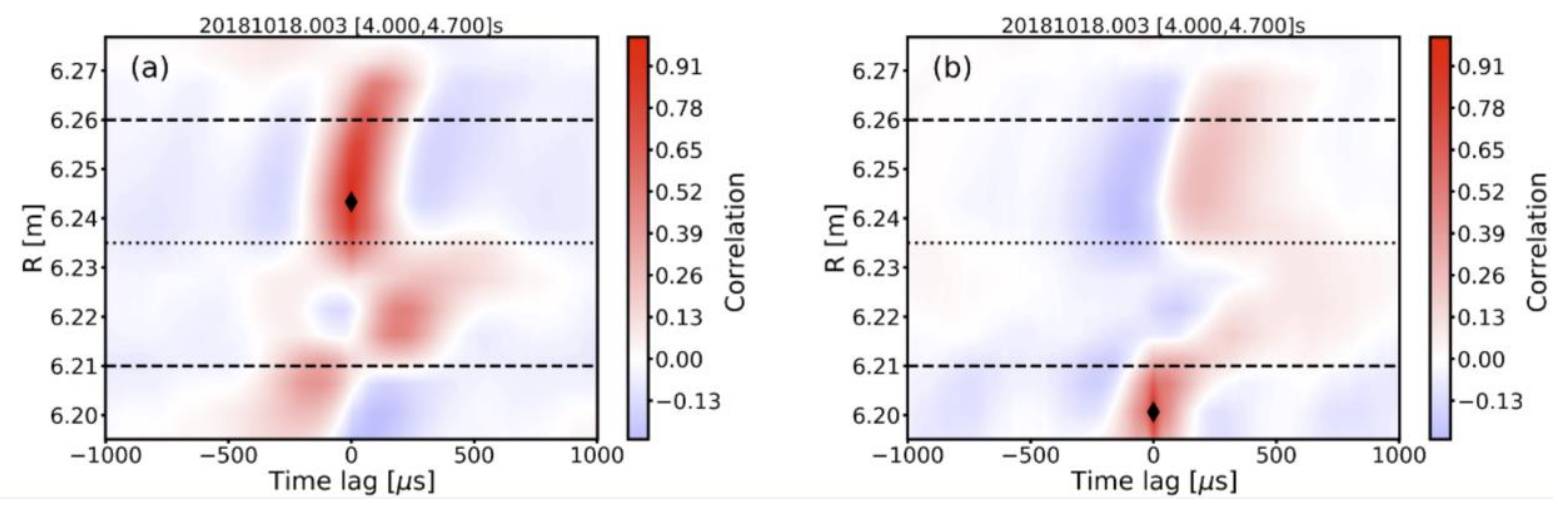

Figure 5. Spatiotemporal correlation function of ABES signals after filtering for $1.6-10 \mathrm{kHz}$ frequency band with first order recursive filter. The diamond symbol marks the reference point in each case. Dashed lines indicate the island separatrices, while the dotted line shows the o-point location. (a) Reference point in island outboard size. (b) Reference point in edge plasma.

In the $10-50 \mathrm{kHz}$ frequency range, where the QC modes are observed, correlation is localized to two narrow regions in the plasma (6.195-6.21 and 6.235-6.255 m). This will be presented in a later section.

To investigate whether the complex correlation structures in Fig. 5. are the results of the magnetic island different configurations were studied as well. In the "high iota" configuration $\left(l_{a}=1.2, \mathrm{FTM}+252,+2.5 \mathrm{~T}\right.$, 
3 MW ECRH, line density $2 \times 10^{19} \mathrm{~m}^{-2}$ ) in one poloidal cross-section 4 islands can be seen, each one is connected to the next after one toroidal turn. The edge island is much narrower, about $2 \mathrm{~cm}$ and the connection length to the divertor plates is shorter. Power spectra and the radial correlation structure of fluctuations in ABES signals are shown in Figure 6. The beam signal was lower due to somewhat reduced beam current, therefore on Figure 6(a) in the outer channels the noise spectrum is dominated by amplifier noise which has increasing power density in the 10-100 kHz range. The broad part of the spectrum is similar to the standard configuration but outside the island the fluctuation power is strongly reduced due to the faster drop of the density. Signature of some QC mode around $10 \mathrm{kHz}$ is also seen in the edge plasma and the island. The radial structure is qualitatively different from the standard configuration. Structures originate inside the island separatrix, similar to the standard configuration, but they propagate radially out with an approximately constant $500 \mathrm{~m} / \mathrm{s}$ radial velocity.

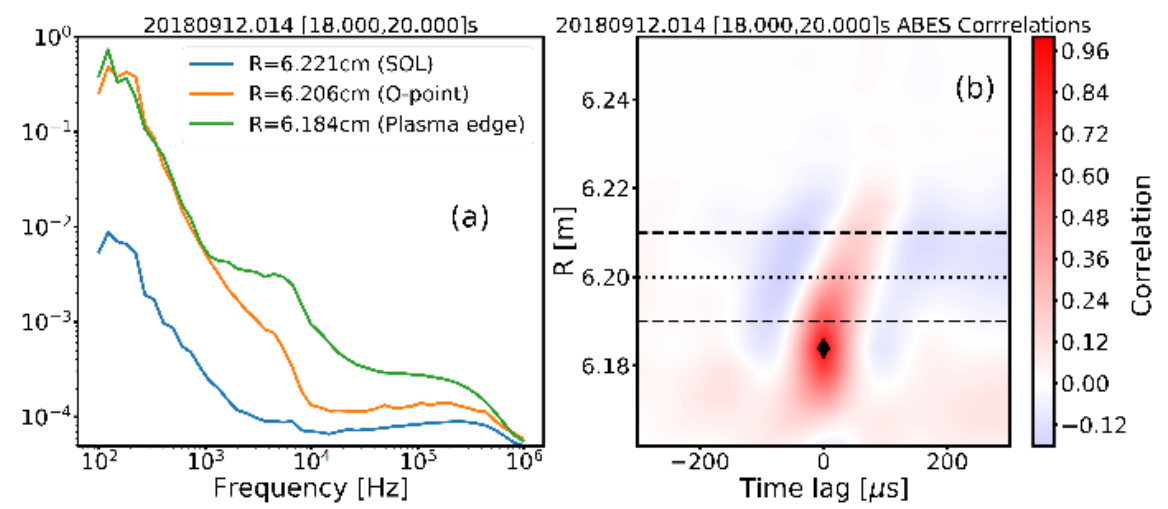

Figure 6. Autopower spectra of selected ABES signals (a) and the spatiotemporal correlation of ABES signals in the edge-island-SOL region for high iota configuration. Filtering of the signals is the same as for Figure 5. The reference point is marked by a diamond. The island separatrices are marked by dashed, the o-point by dotted lines.

The edge islands can also be manipulated in the standard configuration using the control coils located behind each divertor plate. A few discharges were performed in the 2017 experimental campaign by enlarging or shrinking the island radial extent and changing the connection length to the divertor plates. (Discharge 20171207.027 and 20171207.024, EJM-252, -2.5 T, 1.5 MW ECRH, line density $2.5 \times$ $10^{19} \mathrm{~m}^{-2}$, control coil current $-2.5 \mathrm{kA}$ ). In this case the correlation structure seen in Fig. 5. disappears. An example is shown in Fig. 7. In this case the ABES data acquisition system had to operated in a fast "chopping mode" which enable removal of the large background thermal Sodium signal in this campaign[15]. This operation mode limits the sampling rate to $100 \mathrm{kHz}$, therefore spectra are limited to $50 \mathrm{kHz}$. In this case a $\sim 2 \mathrm{~cm}$ radial region is seen with strong correlations without any obvious propagation. Outside this area outward propagating perturbations are seen with about $100 \mathrm{~m} / \mathrm{s}$ radial velocity. The island separatrices are marked at the original location as no detailed field line mapping is available for this configuration. In this configuration the density profile is seen to drop much faster in the plasma edge than in the standard 
configuration and thus on the outboard side of the island the density is much lower than in the standard configuration.

These modified island configurations indicate that the strange correlation structure is a property of the standard configuration of W7-X only.
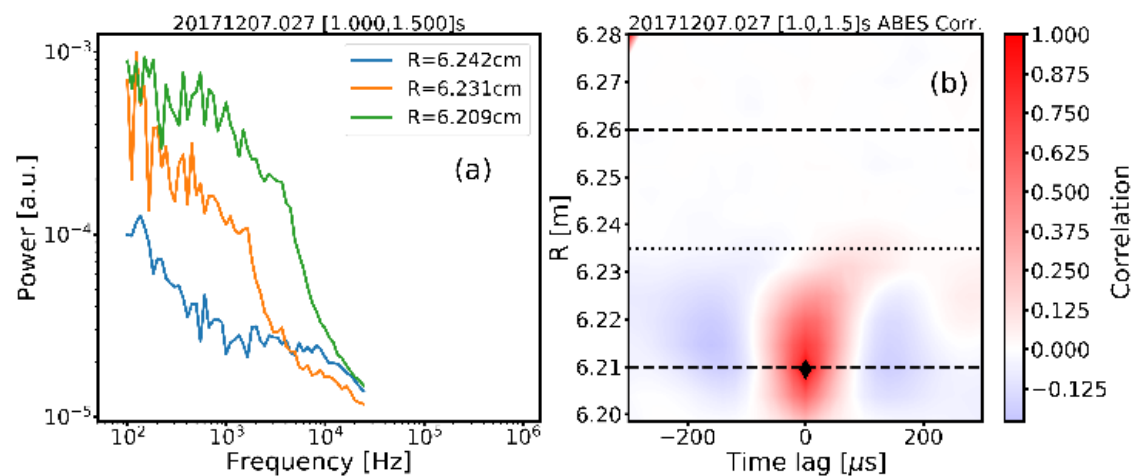

Figure 7. Autopower spectra of selected ABES signals (a) and the spatiotemporal correlation of ABES signals in the edge-island-SOL region when the island is manipulated by the activated control coils. Filtering of the signals is the same as for Figure 5. The reference point is marked by a diamond. The island separatrices in the standard configuration are marked by dashed, the o-point by dotted lines.

\section{Toroidal and poloidal structure}

To check whether the fluctuations observed in the ABES diagnostic correspond to field-aligned structures, correlations using the fast camera measurements were calculated. Unfortunately, in the standard configuration no simultaneous ABES and fast camera measurements are available with high enough signal. Suitable measurements with the somewhat modified island shown in Fig. 7 were available and analysed in detail. In this discharge the rotational transform is not modified significantly as the coils are perturbing the magnetic field locally around the divertors.

The camera was run at $45 \mathrm{kHz}$ frame rate and light filtered around $465 \mathrm{~nm}$ where a Carbon III line is present in the spectrum. The camera image pixels were averaged in $3 \times 3$ boxes and the time evolution of the intensity was correlated with an ABES signal $(\mathrm{R}=6.21)$ as the reference. The resulting data structure has 3 dimensions corresponding to the camera $\mathrm{x}, \mathrm{y}$ pixel coordinate and the correlation time lag. The section of this data at 0 time lag is plotted in Fig. 8(a) as a color coded image. The correlation as a function of time lag is also plotted at four specific locations in the image. Statistically significant positive and negative correlations appear with a few $100 \mu$ s decorrelation time in the image on top of the plasma. The random variance in the correlation functions is indicated by the correlation values towards the edges of the time lag intervals in the correlation functions. The relatively low correlation values are the result of significant uncorrelated broadband noise in the signals. These structures move poloidally to the right when the time lag increases. (That is in the ion diamagnetic direction in this negative magnetic field configuration.) Field lines (in the standard configuration) starting from 
the reference ABES measurement point are also traced and mapped to the camera image. The mapping shows that highest correlation appears in the region connected along field lines to the reference ABES measurement location. Positive and negative correlations are also observed and positive locations seem to be separated by the poloidal distance of the field lines after a toroidal turn. This indicates that the perturbations observed in the edge plasma by the ABES diagnostic are field aligned and possible multiple toroidal turn long. The exact location and shape of the poloidal structure cannot be determined due to inaccuracies in field line geometry and the complicated field line crossings and loops in the image. Lower level but still significant correlations can be observed on other locations as well, resembling a $\sim 1 \mathrm{kHz}$ oscillation. The shape of these correlation structures follows field lines but connections are not direct to the ABES measurement location. These lower frequency structures likely correspond to the ones studied in Ref. [22] and will be investigated in a separate publication.

To be able to better study whether structures are field-aligned and reveal their poloidal structure correlations within camera data were calculated (Figure 8(b)). A toroidal angle was selected in the camera view (115 deg) where most of the flux contours are within the camera region of interest. The flux contour of one of the magnetic islands at this poloidal cross section was mapped to the camera image (solid black line in Figure 8(b)). A point on the inboard side of the island was selected as reference point for the correlation calculations. Positive correlations are observed along field lines mapped around the torus multiple times indicating that filaments can be up to 4 toroidal turn long. On the time resolution of the camera $(45 \mathrm{kHz})$ no time delay is seen between different stripes.

As a summary of camera correlation studies one can state that the edge fluctuations observed by the ABES diagnostic in the modified standard configuration have long filamentary structure with multiple toroidal turn extent, up to $100 \mathrm{~m}$ length. They have a quasi-periodic poloidal structure arising from the proximity of the rotational transform to 1 . Although no suitable ABES-camera correlation measurement is available in the standard configuration it is highly likely that the filament length and poloidal structure is similar in the standard configuration as well. Due to the spatial integration in the camera measurement the exact poloidal structure and the radial localization of the observed poloidal flow direction cannot be determined. 

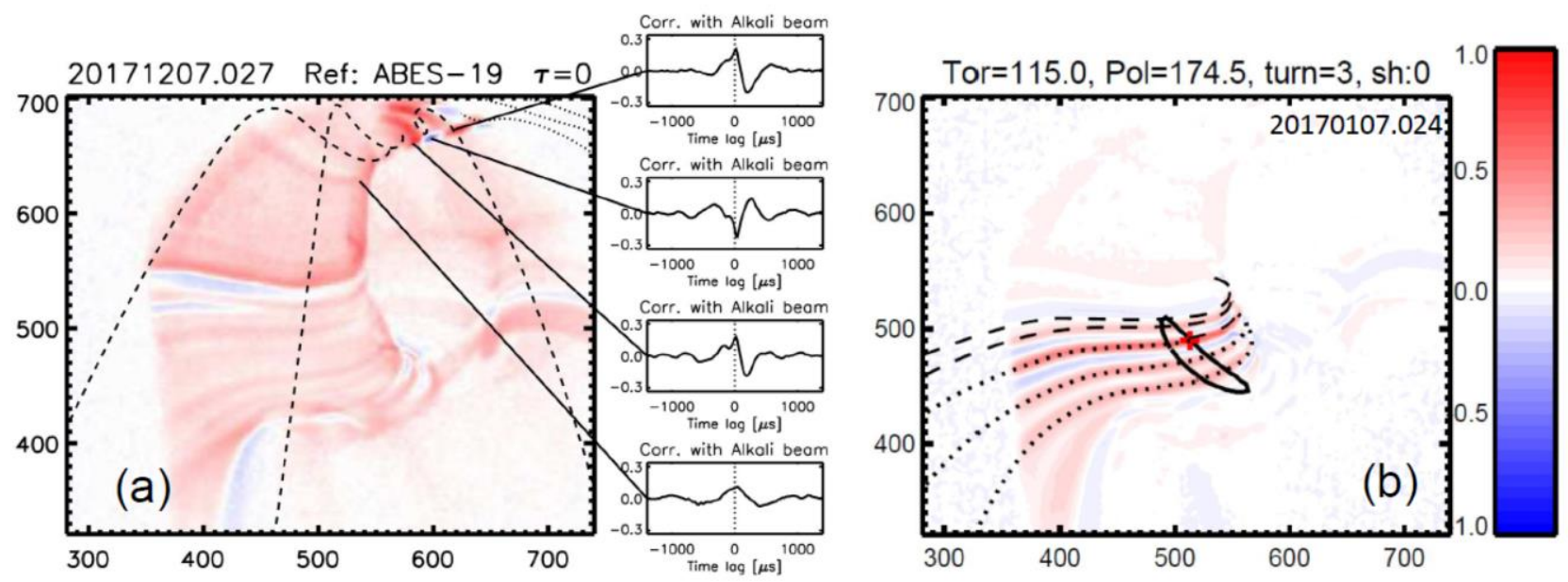

Figure 8. Correlation calculations for discharges with modified islands. (a) Correlation of an ABES edge channel as reference with the camera images. The color scheme is between -0.3 and 0.3, red means positive, blue negative correlation. (b) Correlation using the marked camera point (red cross) as reference. The dashed and dotted lines are field lines started from the reference point and mapped into the camera image in forward and backward toroidal direction, respectively. In (a) the field lines are plotted only in the approx. 75-120 degree toroidal angle, corresponding roughly to the volume observed by the camera. Some field line sections behind walls might also be shown.

\section{Poloidal flow and QC mode}

Local poloidal information on the phenomena was gained by correlation analysis with correlation reflectometry (CR). Instead of the phase the full complex CR signals were used as the phase is affected by $2 \pi$ jumps. The correlation calculation between the real ABES and the complex CR signals yields a complex correlation function of which the amplitude was used. This means CR-ABES correlations are always positive and we look for situations where it is above the expected noise level. Unfortunately, due to an interference of the ABES beam modulation and the CR frequency sweep period, there are joint measurements only at certain radial locations of the CR measurement.

To identify the reflectometry measurement location, the density profile needs to be known along the antenna view direction. As in the island region this information is not known exactly we use correlation analysis between CR and ABES to help better localize the CR measurement.

The QC mode shown in Figure 4 is discussed first. Figure 9(a) and (c) show the correlation between reflectometry antenna B (uppermost) and the ABES channels around the edge island when the signals are filtered for the QC frequency $(10-30 \mathrm{kHz})$ band. As the reflectometry frequency was hopped with $20 \mathrm{~ms}$ step time each figure corresponds to correlations calculated in a $18 \mathrm{~ms}$ interval when the CR frequency is 
fixed. In (a) CR measured on the outboard side of the island, while in (c) it probed the edge plasma. At these two locations significant correlation appears with ABES around the CR measurement locations. The correlation calculations in (b) and (d) show the same correlation structure within the ABES signals when the reference channel is placed to the location where maximum correlation is seen with CR. Such correlation exists only when CR measures in the vicinity of the above radial locations indicating that the QC mode is localized to these two regions. No correlation exists between the two regions either. This observed ABES$\mathrm{CR}$ correlation also localizes the $\mathrm{CR}$ measurement relative to ABES without knowledge of the density profile.
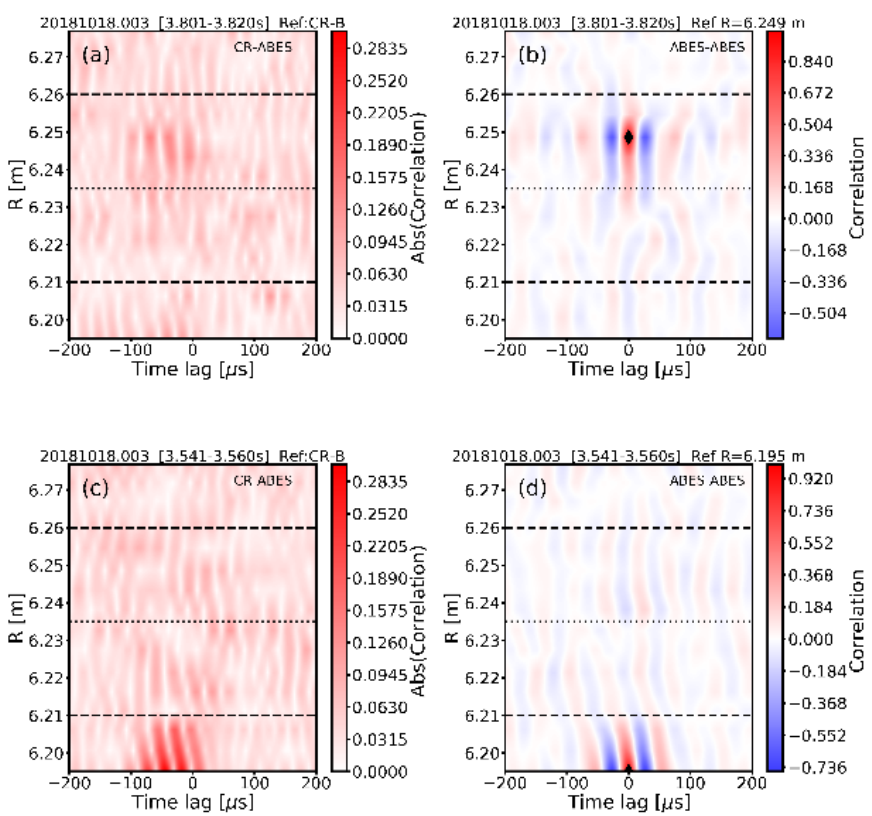

Figure 9. Correlation of ABES channels measuring in the island and edge region with reflectometer antenna $B$ ( $a$ and $c$ ) and with selected ABES channels ( $b$ and $d)$. In (a) reflectometer measured on the outboard side of the island while in (c) in the edge plasma. In plot(b) and (d) the reference signal is chosen where the correlation is maximum with reflectometry. The signals were filtered for $10-30 \mathrm{kHz}$ frequency band. The dashed lines mark the island separatrices, the dotted line is the o-point from the vacuum field calculation. The diamond symbol marks the reference position for the ABES correlations.

From the phase shift between poloidally offset CR antenna signals[19] and the oscillation period, the poloidal flow velocity and wavelength of the two QC modes are $\mathrm{v}=6.6 \mathrm{~km} / \mathrm{s}$ (downward, electron diamagnetic drift direction), $\lambda=34 \mathrm{~cm}$ at $\mathrm{R}=6.2 \mathrm{~m}$ and $\mathrm{v}=4 \mathrm{~km} / \mathrm{s}$ (upward), $\lambda=19 \mathrm{~cm}$ at $\mathrm{R}=6.25 \mathrm{~m}$. Although the $\mathrm{CR}$ and $\mathrm{ABES}$ diagnostics run from a common sampling clock, the relative delay in their start trigger was not checked. As a result, the detailed phase relationship between the two diagnostics cannot be studied.

From the poloidal propagation of the broadband turbulence across CR antennae, it is seen that the poloidal flow velocity changes sign at the inner separatrix $(6.21 \mathrm{~m})$ in agreement with the finding in Ref [19]. The tilt in the correlation in the edge plasma is most probably the result of tilt in the radial-poloidal plane[25]. If the tilt was caused by the sheared flow, then outward apparent propagation would be seen. This is in contrast to the tilt direction of structures at the plasma edge in Fig 9 (c) and (d). 
The CR-ABES correlation in the $1-10 \mathrm{kHz}$ frequency band is less consistent. In some time intervals strong correlation is detected between ABES and CR signals with 0 time lag which point to the existence of structures with a poloidal size reaching $10 \mathrm{~cm}$. At present it is not clear whether these are the filamentary structures, some other phenomena at the lower part of the frequency band or maybe multiple toroidal turns of the same structure. 


\section{Discussion}

The results presented in this paper indicate that density fluctuations in the $1-10 \mathrm{kHz}$ frequency range in the edge of the standard configuration plasma in the W7-X device are field-aligned perturbations. Their toroidal length in the edge plasma is multiple toroidal turns, in a somewhat special measurement video cameras detected 4 toroidal turns. In this measurement the time lag between turns is less than $22 \mu$ s (the camera time resolution), much shorter than the ion transit time on one toroidal turn. ( $200 \mu \mathrm{s}$ at the measured $\sim 100 \mathrm{eV}$ edge ion temperature.) The perturbations travel radially out but disappear from the view of the alkali BES diagnostic just after crossing the island separatrix. The separatrix position was verified by observation of a shear layer in the poloidal flow velocity[19], the filaments disappear slighly outside the shear layer. They appear again about $200 \mu \mathrm{s}$ later on the outboard side of the island, close to the o-point. This behaviour is observed only in the standard configuration. From the reappearance point they propagate radially out with $400 \mathrm{~m} / \mathrm{s}$ velocity which seems to decrease outside the island. In this region the connection length decreases dramatically[20], and the filaments might enter the sheath limited regime where their polarization and their resulting ExB velocity is reduced. Similar slowing down is observed in the high iota configuration radially outside the narrow edge island.

Several possibilities can be considered for the propagation of the filaments from the island inner separatrix to the outboard side of the island. If toroidal propagation is considered, the observed $\sim 200 \mu$ s time delay is too short for ions to travel multiple times around the torus following field lines. (See Figure 10.) On the other hand the transit time for electrons over 3-5 toroidal turns is only in the 30-50 $\mu$ s range, too short for the observed time delay. Another possibility would be that the perturbations arriving from the edge plasma are caught by the poloidal flow in the island and moved around to the outboard side. The $0.5-1 \mathrm{~m}$ path length and the few $\mathrm{km} / \mathrm{s}$ poloidal flow velocity would indeed place the time lag into the observed magnitude, and also Figure 5 hints to some rotating structure in the 6.21-6.235 m region. However, there are two problems with this explanation. One is that the perturbation trajectory would not follow a flux surface, the second is that it does not explain the origin of the perturbation going out from the island. An alternative explanation might be that filament generation in the two radial regions with significant density (and possibly pressure) gradient is in some way synchronized by perturbations traveling around the island with the poloidal flow. Two gradient regions are observed only in the standard configuration, and similarly the complex radial propagation is only observed in this case.

The location of these regions is illustrated relative to the measured density profile on Fig. 10. Additionally to the low frequency blob-like features, a quasi-coherent mode is also observed in the $10-30 \mathrm{kHz}$ frequency range close to the two high-gradient regions. The poloidal wavelength of the QC mode at both locations is about 20-30 cm, close to the poloidal displacement of a field line after one toroidal turn. The magnetic geometry might have a role in setting this poloidal wavelength of the QC modes. The relation between the 
QC mode and the filaments is not clear although their relative radial location might hint to filament generation from a coherent mode as e.g. in the case of ELMs.

\section{Comparison with filaments in other machines}

Filamentary structures seen at the edge of W7-X plasmas show several similar features to filaments (blobs) observed in tokamaks and other toroidal devices[1]. Their radial size is in the $\mathrm{cm}$ range, similar to larger blobs in tokamaks. It has to be noted that the three diagnostics used in this study have $1-2 \mathrm{~cm}$ spatial resolution, therefore smaller blobs cannot be detected. Their radial velocity is directed outward with a few $100 \mathrm{~m} / \mathrm{s}$, comparable to velocities in tokamaks. In the toroidal direction they follow magnetic field lines over multiple toroidal turns in a way that the time lag of the perturbation along the field lines is significantly shorter than the transit time of ions. This is in good agreement with tokamak measurements[4][26][27] and indicates that the perturbation has $\mathrm{k} \| \approx 0$. The considerably longer toroidal length than in tokamaks is in good agreement with the much longer connection length to the divertors (up to $1 \mathrm{~km}$ ) even at the inner side of the edge island.

At present the poloidal size if the filaments in W7-X is not clear. Camera data in Fig 8. indicate few $\mathrm{cm}$ poloidal size which would be consistent with tokamak observations. On the other hand, occasionally correlation is observed on $10 \mathrm{~cm}$ poloidal distance between the ABES and CR diagnostic. This might be the result of insufficient statistics, multiple toroidal turn long filaments or the appearance of lower frequency larger poloidal size events.

Another significant difference to tokamak blobs is the absent of intermittency in the data, in a single signal it is difficult to identify individual events. Looking at multiple signals at the same time individual events with both positive and negative perturbation can still be identified (see Fig. 3.) The reason for negative perturbations might be that perturbations temporally overlap and outward propagating negative perturbations are actually caused by a temporarily lower number of perturbations.

Due to the above similarities and differences at present it cannot be stated whether the filaments observed in W7-X are a variation of the familiar tokamak blobs or should be considered as a different phenomenon.

\section{Role of filaments in edge transport}

The filamentary perturbations described in the present paper originate from the edge plasma and travel through the edge island to the far SOL, therefore they should play some role in the transport in these regions. At present only a very crude order of magnitude estimate can be made of the particle loss through them by observing the density profile change in the island at large events and making assumptions on the filament frequency. This puts the global particle time into the 1-10 s range, 1-2 orders of magnitude longer than the 
particle confinement time measured by impurity injection, indicating that these filaments do not play an important role in global particle transport.

In contrast to this in the island and far SOL region filaments might play a role in transport. Close to the island o-point the density profile is flat or in some cases even strongly peaked[15][17]. Although the area around the o-point is a closed flux tube without connection to the divertor plates, an elevated density would require some anomalous transport mechanism balancing the classical B-perpendicular transport out from the island. One such mechanism might be filament ejection into the island as seen by correlation analysis. The time evolution of the density in the vicinity of the o-point indeed indicates fast jumps both up and down, which might be caused by filaments ejected into and out from the island, respectively.

One more observation should be mentioned at this point. Low frequency (200-400 Hz) quasi periodic oscillations in the edge plasma are often observed in W7-X[21]. In the high-iota configuration the filament activity was clearly seen to be modulated in this cycle, the lowest activity is observed when the density profile is the steepest. This might indicate either that filaments play a role in setting the edge density profile or filament activity is dependent on the density profile.
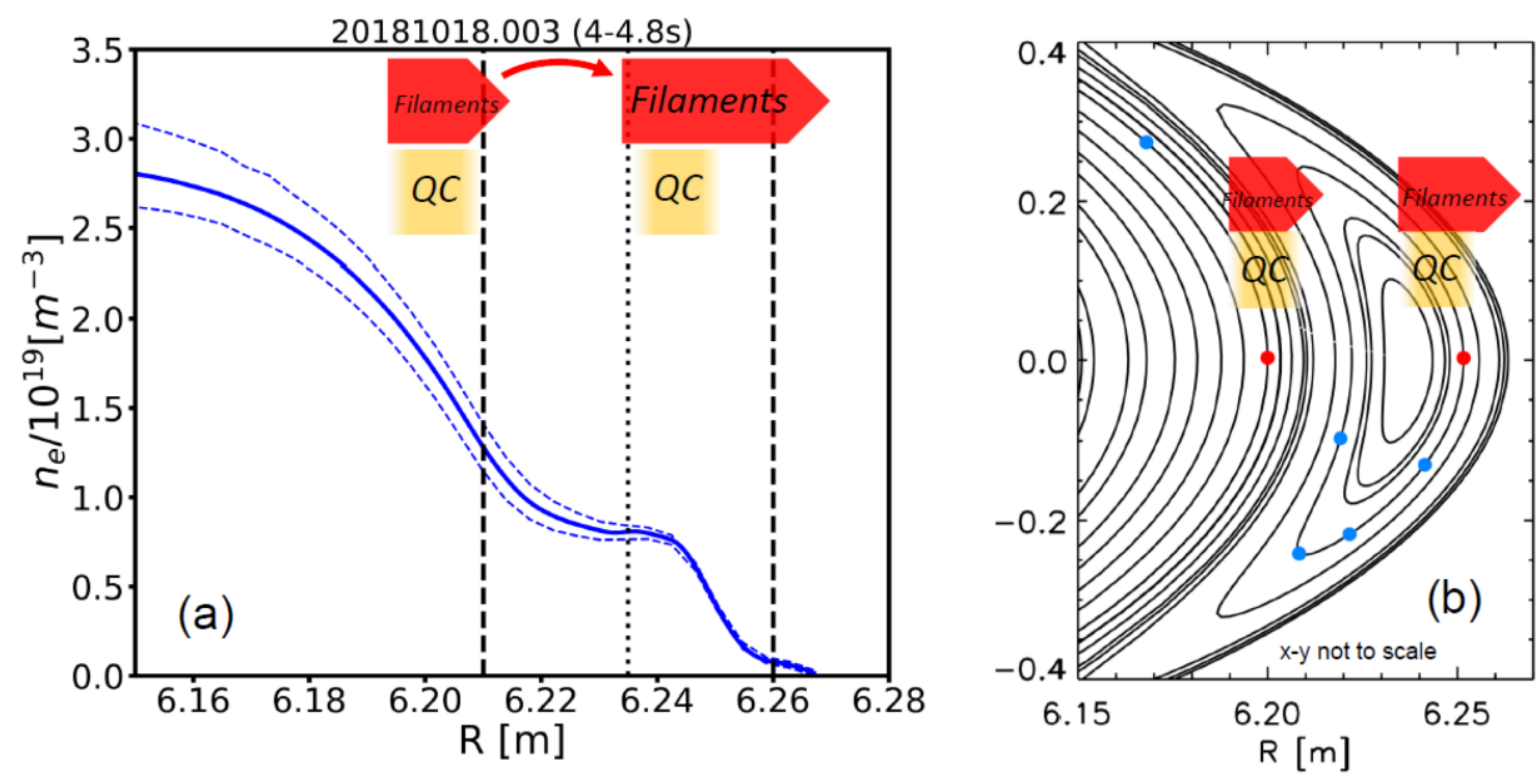

Figure 10. (a) Density profile and its estimated error reconstructed from ABES data[17] in the standard configuration. (b) Mapping of points on the equatorial plane (red dots) in the QC mode radial regions along field lines after multiple toroidal turns in one toroidal direction (blue dots). The approximate regions of the blobs and QC modes are illustrated on both subplots.

\section{Conclusions}

Field-aligned plasma filaments are regularly observed in the edge of W7-X plasmas. A multi-diagnostic analysis reveals that in the standard configuration, when the plasma is bounded by a natural island chain, multiple toroidal turn long filaments are ejected in a correlated way from the edge plasma into the island 
and from the outboard side of the island to the far SOL, but they are not seen to cross the island. The correlation mechanism between the two regions is not revealed yet. Close to both radial ranges for filament generation, a quasi-coherent mode with long $(20-30 \mathrm{~cm})$ poloidal wavelength was detected, which might be somehow related to filament generation. A scheme for the radial location of these structures relative to the density profile and magnetic configuration is shown in Figure 10.

Although these filaments are likely not playing a role in the plasma global particle transport they appear to play a significant role in setting the density in the o-point of the island and thus might play a role in setting island divertor operating parameters.

Some features of these filaments fit well to properties of filaments observed in tokamaks: radial propagation velocity, $\mathrm{k} \| \approx 0$. On the other hand, there are significant differences as well, most notably lack of clear intermittency, therefore at present it cannot be stated whether the filaments observed in W7-X are a variation of the familiar tokamak blobs or should be considered as a different phenomenon.

\section{Acknowledgements}

This work was carried out within the framework of the EUROfusion Consortium and has received funding from the Euratom research and training programme 2014-2018 and 2019-2020 under grant agreement No 633053. The views and opinions expressed herein do not necessarily reflect those of the European Commission. Invaluable comments from J.L. Terry are highly acknowledged.

\section{References}

[1] D’Ippolito D A, Myra, J R and Zweben S J 2011 Phys. Plasmas 18060501

[2] Carralero D, Manz P, Aho-Mantila L, Birkenmeier G, Brix M. Groth M, Müller HW, Stroth U, Vianello N, Wolfrum E, ASDEX Upgrade team, JET Contributors and EUROfusion MST1 Team 2015 Phys. Rev. Lett. 115215002

[3] Harrison J R, G. Fishpool M, Thornton AJ, Walkden N R and the MAST team 2015 Phys. Plasmas S22 092508

[4] Walkden N R, Harrison J, Silburn S A, Farley T, Henderson SS, Kirk A, Militello F, Thornton A and the MAST Team 2017 Nucl. Fusion 57126028

[5] Militello F, Walkden N R, Farley T, Gracias W A, Olsen J, Riva F, Easy L, Fedorczak N, Lupelli I, Madsen J, Nielsen A H, Ricci P, Tamain P and Young J 2016 Plasma Phys. Control. Fusion 58105002

[6] Endler M, Giannone L, McCormick K, Niedermeyer H, Rudyj A, Theimer G, Tsois N, Zoletnik S, ASDEX Team and W7-AS Team 1994, Physica Scripta 51610

[7] Wolf R C, et al 2017 Nucl. Fusion 57102020

[8] Klinger T, et al 2019 Nucl. Fusion 59112004 
[9] Feng Y, Kobayashi M, Lunt T and Reiter D 2011 Plasma Phys. Control. Fusion 53024009

[10] Sunn Pedersen T, et al 2019 Nucl. Fusion 59096014

[11] Shanahan B, Dudson B and Hill P 2018 IOP Conf. Series: Journal of Physics: Conf. Series 1125 012018

[12] Kocsis G, Alonso A, Biedermann C, Cseh G, Dinklage A, Grulke O, Jakubowski M, König R, Krychowiak M, Otte M, Sunn Pedersen T, Szepesi T, Wenzel U, Xanthopoulos P, Zoletnik S and the W7-X Team 2017 44th EPS Conf. on Plasma Phys. ECA Vol 41F O2.102

[13] Kocsis G, Baross T, Biedermann C, Bodnar G, Cseh G, Ilkei T, König, Otte M, Szabolics T, Szepesi T, Zoletnik S 2015 Fus. Eng. Des. 96-97 707

[14] Zoletnik S, Biedermann C, Cseh G, Kocsis G, König R, Szabolics T, Szepesi T and Wendelstein 7-X Team 2018 Rev. Sci. Instrum. 89013502

[15] Zoletnik S, Anda G, Aradi M, Asztalos O, Bató S, Bencze A, Berta M, Demeter G, Dunai D, Hacek P, Hegedűs S, Hu G H, Krizsanóczi T, Lampert M, Nagy D, Németh J, Otte M, Petravich G, Pokol G I, Réfy D, Tál B, Vécsei M and W7-X Team 2018 Rev. Sci. Instrum. 89 10D107

[16] Anda G, Dunai D, Lampert M, Krizsanóczi T, Németh J, Bató, S, Nam Y U, Hu G H and S. Zoletnik 2018 Rev. Sci. Instrum. 89013503

[17] Vécsei M, Anda G, Asztalos O, Brunner K J, Dunai D, Hirsch M, Knauer J, Otte M, Pokol G I, Zoletnik S and the W7-X team 2019 46th EPS Conf. on Plasma Phys. P4.1021

[18] Windisch T, Krämer-Flecken A, Velasco J L, Könies A, Nührenberg C, Grulke O, Klinger T and the W7-X team 2017 Plasma Phys. Control. Fusion 59105002

[19] Krämer-Flecken A, Han X, Windisch T, Cosfeld J, Drews P, Fuchert G, Geiger J, Grulke O, Killer C, Knieps A, Liang Y, Liu S, Rack M and the W7-X team, 2019 Plasma Phys. Control. Fusion 201861 054003

[20] Liu S C, Liang Y, Drews P, Killer C, Knieps A, Xu G S, Wang H Q, Yan N, Han X, Höschen D, Krämer-Flecken A, Nicolai D, Satheeswaran G, Hammond K, Cai1 J Q, Charl A, Cosfeld J, Fuchert G, Gao Y, Geiger J, Grulke O, Henkel M, Hirsch M, Hoefel U, König R, Li Y, Neubauer O, Pasch E, Rahbarnia K, Rack M, Sandri N, Sereda S, Schweer B, Wang E H, Xu S, Gao X and W7-X Team 2019 Nucl. Fusion 59066001

[21] Wurden G A, Ballinger S, Bozhenkov S, Brandt C, Buttenschoen B, Endler M, Freundt S, HammondK, Hirsch M, Hoefel U, Killer C, Kocsis G, Kornejew P, Krychowiak M, Lazerson S, Rahbarnia K, Szepesi T, Windisch T, Winters V and the W7-X Team 2018 45th EPS Conf. on Plasma Phys. ECA Vol 42A P5.1077

[22] Ballinger S B, Terry J L, Baek S G, Tang K, von Stechow A, Killer C, Nicolai D, Satheeswaran G, Drews P, Grulke O and W7-X Team 2018 Nucl. Mat. Energy 17269

[23] Lampert M, Zoletnik S, Bak J G, Nam Y U and KSTAR Team 2018 Phys. Plasmas 25042507 
[24] Willensdorfer M, Birkenmeier G, Fischer R, Laggner F M, Wolfrum E, Veres G, Aumayr F, Carralero D, Guimarais L, Kurzan B and the ASDEX Upgrade Team 2014 Plasma Phys. Control. Fusion 56025008

[25] Fedorczak O, Manz P, Thakur SC, Xu M, Tynan GR, Xu GS, Liu, SC 2012 Phys. Plasmas 19122302 (2012)

[26] Grulke O, Terry J L, Cziegler I, LaBombard B and Garcia O E 2014 Nucl. Fusion 54043012

[27] Thomsen H, Endler M, Bleuel J, Chankin A V, Erents S K, Matthews G F 2002 Phys. Plasmas 9 1233 